\title{
Current Health News
}

Exercise alters brain chemistry to protect aging synapses

Date: January 7, 2022

Source: University of California - San Francisco

When elderly people stay active, their brains have more of a class of proteins that enhances the connections between neurons to maintain healthy cognition, a new study has found. This protective impact was found even in people whose brains at autopsy were riddled with toxic proteins associated with Alzheimer's and other neurodegenerative diseases.

No more annual flu shot? New target for universal influenza vaccine

Date: December 23, 2021

Source: Scripps Research Institute

Summary: A new antibody discovered in the blood of some people vaccinated against or infected with influenza can recognize a broad variety of flu viruses.

Can a dangerous microbe offer a new way to silence pain?

Date: December 20, 2021

Source: Harvard Medical School

Summary: A new study shows that a toxin from the microbe that causes anthrax can silence multiple types of pain in mice. Researchers found the toxin targets pain-sensing cells to alter signaling and block pain. Building on that finding, researchers engineered an anthrax protein vehicle to deliver different types of treatments into pain receptors and modulate nervecell function. The findings can inform the design of therapies that selectively target pain-sensing fibers without the widespread systemic effects of opioids and other pain killers.

Shark antibody-like proteins neutralize COVID19 virus, help prepare for future coronaviruses

Date: December 17, 2021

Source: University of Wisconsin-Madison
Summary: Small, unique antibody-like proteins known as VNARs -- derived from the immune systems of sharks -- can prevent the virus that causes COVID-19, its variants, and related coronaviruses from infecting human cells, according to a new study.

\section{Cellular receptors identified for eastern equine} encephalitis

Date: January 14, 2022

Source: Harvard Medical School

Summary: A new study has identified a set of cellular receptors for at least three related alpha viruses shared across mosquitoes, humans, and animals that host the virus.

Repeated exposure to major disasters has longterm mental health impacts

Date: January 16, 2022

Source: Texas A\&M University

Summary: Repeated exposure to major disasters does not make people mentally stronger, a recent study found: individuals who have been repeatedly exposed to major disasters show a reduction in mental health scores.

\section{Being in space destroys more red blood cells}

Date: January 14, 2022

Source: The Ottawa Hospital

Summary: A world-first study has revealed how space travel can cause lower red blood cell counts, known as space anemia. Analysis of 14 astronauts showed their bodies destroyed 54 percent more red blood cells in space than they normally would on Earth, according to a new study.

\section{Biologists pinpoint key factor in immune system response to viral infection}

Date: January 14, 2022

Source: University of California - San Diego

Summary: Researchers studying how small worms defend themselves against pathogens have discovered a gene that acts as a first-line response against 
infection. They identified 'ZIP-1' as a centralized hub for immune response, a finding could have implications for understanding human immunity against viruses.

Like our social media feeds, our brains take a little while to update

Date: January 13, 2022

Source: University of California - Berkeley

Summary: Our brains are constantly uploading rich visual stimuli. But instead of seeing the latest image in real time, we actually see earlier versions because our brain's refresh time is about 15 seconds. The findings add to a growing body of research about the mechanism behind the 'continuity field,' a function of perception in which our brain merges what we see to give us a sense of visual stability.

\section{Novel treatment target for heart disease found in the blood vessel wall}

Date: January 14, 2022

Source: Medical College of Georgia at Augusta University

Summary: A molecule of RNA called CARMN has been found in abundance in the healthy smooth muscle cells that help give our blood vessels strength and flexibility, and distinctly decreased in vascular diseases like atherosclerosis, a major cause of heart attack and stroke, scientists report.

\section{Reference: sciencedaily.com}

\section{Common cold may shield against COVID}

Tuesday, January 11, 2022

Vaccination is still the best way to protect someone from COVID-19, but new research suggests that immune system activation of T-cells by common colds may offer some cross-protection. The study might also provide a blueprint for a secondgeneration, universal vaccine that could prevent infection from current and future variants, the research team said.
CDC updates mask guidelines - cloth masks least effective

January 16, 2022 -- The U.S. Centers for Disease Control and Prevention has updated its guidance on face masks, saying loosely woven cloth masks offer the least protection against COVID-19 and N95 and KN95 masks offer the most. The CDC update doesn't come out and say Americans should avoid cloth masks but clarifies that some kinds of masks work better than others.

Reference: cnn.com/health

\section{To stop blood cancer, target the bone}

Date: January 19, 2022

Source: Columbia University Irving Medical Center

Summary: New research suggests that targeting neighboring bone cells may be a better strategy than targeting malignant stem cells to treat acute myeloid leukemia.

Artificial pancreas proves 'life-changing' for very young children with type 1 diabetes and their families

Date: January 20, 2022

Source: University of Cambridge

Summary: An artificial pancreas is helping protect very young children with type 1 diabetes at a particularly vulnerable time of their lives. A study published today found that it is both safe to use and more effective at managing their blood sugar levels than current technology.

You can feel this acid when you work out. Now it may increase knowledge of cancer medicine

Date: January 20, 2022

Source: University of Copenhagen - The Faculty of Health and Medical Sciences

Summary: New research shows that specific enzymes can remove lactic acid marks. This finding may increase our understanding of cancer medicine and how physical exercise, among other things, can affect human epigenetics. 


\section{Supplement appears to boost muscle, mitochondria health}

Date: January 20, 2022

Source: University of Washington School of Medicine/UW Medicine

Summary: An oral supplement intended to stimulate a natural body process appears to promote muscle endurance and mitochondrial health in humans. New research suggests that the supplement, urolithin A, may help improve or prolong muscle activity in people who are aging or who have diseases that make exercise difficult.

COVID-19 vaccines do not cause infertility, study finds

Date: January 20, 2022

Source: Boston University School of Medicine

Summary: New findings indicate that COVID-19 vaccination does not impair fertility, but males who become infected by the coronavirus may experience short-term reduced fertility.

\section{An all-in-one approach to diabetes treatment}

Date: January 20, 2022

Source: Massachusetts Institute of Technology

Summary: A team of researchers has come up with a new approach to streamline the process that people with diabetes use to estimate the carbohydrate content of a meal, draw blood to measure current blood glucose levels, and calculate and deliver the correct insulin dose.

\section{Mapping dementia-linked protein interactions yields potential new treatment targets}

Date: January 20, 2022

Source: Weill Cornell Medicine

Summary: By mapping all the protein interactions of a dementia-linked protein in the brain called Tau, a team of investigators has created a road map for identifying potential new treatment targets for Alzheimer's disease and related dementia.
Newly discovered DNA repair mechanisms point to potential therapy targets for cancer and neurodegenerative diseases

Date: January 20, 2022

Source: Massachusetts General Hospital

Summary: Faulty DNA damage repair can lead to many types of cancer, neurodegenerative diseases, and other serious disorders. Investigators have developed high-throughput microscopy and machine learning systems that can identify and classify DNA repair factors. The investigators have identified nine previously unknown factors involved in the process of cellular DNA repair.

\section{TV watching linked with potentially fatal blood clots}

Date: January 19, 2022

Source: European Society of Cardiology

Summary: Take breaks when binge-watching TV to avoid blood clots, say scientists. The warning comes as a study reports that watching TV for four hours a day or more is associated with a $35 \%$ higher risk of blood clots compared with fewer than 2.5 hours.

\section{'Decoy' protein works against multiple SARS- CoV-2 variants, researchers report}

Date: January 19, 2022

Source: University of Illinois Chicago

Summary: A drug treatment that acts as a decoy against SARS-CoV-2 was highly effective at preventing death and lung damage in humanized animal models of severe COVID-19 disease. The study suggests that the drug has the potential to treat COVID-19 patients, including those who are infected with aggressive SARS-CoV-2 variants.

For Glioma patients, a mutated gene may open the door to new treatment options

Date: January 19, 2022

Source: Michigan Medicine - University of Michigan Summary: A mutated gene affects growth of brain tumor cells in young adults, indicating sensitivity to a new treatment strategy, a team of researchers has discovered. These findings present possibilities for 
more effective therapies for glioma patients with this gene mutation.

\section{The role of ribosomes in age-related diseases}

Date: January 19, 2022

Source: Stanford University

Summary: Research finds that the cellular assembly line that produces proteins can stall with age, triggering a snowball effect that increases the output of misfolded proteins. In humans, clumps of misfolded proteins contribute to age-linked Alzheimer's and Parkinson's diseases.

\section{Magnesium is essential for the immune system, including in the fight against cancer}

Date: January 19, 2022

Source: University of Basel

Summary: The level of magnesium in the blood is an important factor in the immune system's ability to tackle pathogens and cancer cells. Researchers have reported that $\mathrm{T}$ cells need a sufficient quantity of magnesium in order to operate efficiently. Their findings may have important implications for cancer patients.

\section{Tracking the lifespan and myriad functions of mRNA}

Date: January 19, 2022

Source: Yale University

Summary: New studies shed light on how mRNAs are born and how they regulate production of proteins inside of our cells once they reach maturity. The findings have implications not only for achieving effective doses for new vaccines, but for helping determine the biological roots of many cancers and diseases.

Reference: www.sciencedaily.com

\section{Information collected and compiled by}

Md. Akbar Hossain

ASA University (ASAUB)

Shyamoli, Mohammadpur

Dhaka-1207, Bangladesh 